\title{
Corneal Neurotization and Novel Medical Therapies for Neurotrophic Keratopathy
}

\author{
Tatiana R. Rosenblatt ${ }^{1}$ (D) $\cdot$ Connie M. Sears ${ }^{1} \cdot$ Ji Kwan Park ${ }^{2} \cdot$ Andrea Lora Kossler $^{1}$ (D)
}

Published online: 23 September 2020

(C) The Author(s) 2020

\begin{abstract}
Purpose of Review Neurotrophic keratopathy (NK) is a degenerative corneal disease characterized by decreased corneal sensibility and impaired corneal healing. In this article, we review surgical techniques for corneal neurotization (CN) and novel medical therapies for the treatment of NK.

Recent Findings In recent decades, there has been a paradigm shift in the treatment strategies for NK. New minimally invasive direct and indirect $\mathrm{CN}$ approaches have demonstrated efficacy at improving best-corrected visual acuity and central corneal sensation while decreasing surgical morbidity. In addition, several targeted medical therapies, such as recombinant human nerve growth factor (rhNGF), regenerating agents (RGTA), and nicergoline, have shown promise in improving corneal epithelial healing. Of these options, cenegermin (Oxervate®, Dompé), a topical biologic medication, has emerged as an approved medical treatment for moderate to severe NK.

Summary NK is a challenging condition caused by alterations in corneal nerves, leading to impairment in sensory and trophic function with subsequent breakdown of the cornea. Conventional therapy for NK depends on the severity of disease and focuses primarily on protecting the ocular surface. In recent years, numerous $\mathrm{CN}$ techniques and novel medical treatments have been developed that aim to restore proper corneal innervation and promote ocular surface healing. Further studies are needed to better understand the long-term efficacy of these treatment options, their target populations, and the potential synergistic efficacy of combined medical and surgical treatments.
\end{abstract}

Keywords Corneal neurotization · Corneal neurotization · Neurotrophic keratopathy · Cenegermin · Corneal epithelial defect · Neurotrophic keratitis · Nerve growth factor

\section{Introduction}

The cornea is the most densely innervated tissue in the body, making it 300-600 times more sensitive than the skin [1-3]. The dense innervation of the cornea contributes to ocular sensations such as dryness, discomfort, and pain, as well as

This article is part of the Topical Collection on Regenerative Medicine in Ophthalmology

Andrea Lora Kossler

akossler@stanford.edu

1 Department of Ophthalmology, Byers Eye Institute, Stanford University School of Medicine, 2452 Watson Court, Palo Alto, CA 94303-3216, USA

2 Department of Ophthalmology, Loma Linda Eye Institute, Loma Linda University School of Medicine, 11370 Anderson St. \#1800, Loma Linda, CA 92354, USA functions such as the blink and tearing reflexes, and corneal wound healing. Thus, intact corneal innervation is vital for maintaining the structural and functional integrity of the ocular surface.

A symbiotic relationship exists between the corneal epithelium and the nerves that innervate it. Corneal nerves secrete substances such as substance $\mathrm{P}$ and calcitonin gene-related peptide, which promote proliferation of the corneal epithelium and play a key role in wound healing of the ocular surface [3]. Meanwhile, corneal epithelial cells secrete factors that promote the health and survival of the corneal nerves, such as neurotransmitters, neuropeptides, neurotrophins, and growth factors $[1,3,4]$. This dynamic relationship serves to maintain the integrity and function of the ocular surface, which in turn plays a vital role in ocular health by reducing dry eye symptoms, protecting the eye from trauma and infection, promoting eye wound healing, and minimizing visual distortion to improve visual acuity [1]. Without proper corneal innervation, 
the corneal epithelium is vulnerable to breakdown and eventual corneal opacification, which is a leading cause of blindness [2]. Therefore, it is crucial that improper corneal nerve function be effectively restored.

The cornea is innervated by the ophthalmic division of the trigeminal nerve (cranial nerve V). Approximately 50-450 sensory trigeminal neurons emanate from the ophthalmic branch and terminate as free nerve endings within the corneal epithelium $[2,5]$. Nerve fibers extend from trigeminal ganglion cells and branch into nerve bundles that lay uniformly around the corneoscleral limbus, forming the limbal plexus. From the limbal plexus arise 33-71 stromal nerve bundles that perforate Bowman's layer to form dense nerve plexi beneath the basal epithelial cells, with an increasing number of nerve divisions as the distance from the corneoscleral junction increases [6]. Stromal nerves are both myelinated and unmyelinated. Some of them terminate in free nerve endings, while others innervate keratocytes, contributing to the reciprocal relationship between the ocular tissues and their nerves [2]. In addition to innervating the cornea, the trigeminal nerve serves as the afferent limb of two important ocular reflex arcs via the facial nerve: the blink response (motor) and the secretion of goblet cells, lacrimal glands, and meibomian glands (autonomic), which play key roles in overall eye function [7]. Thus, trigeminal nerve damage or dysfunction, particularly of the ophthalmic branch, can lead to devastating consequences for corneal health.

\section{Neurotrophic Keratopathy}

Neurotrophic keratopathy (NK) is a rare degenerative corneal condition with a prevalence of 1.6 to 4.2 per 10,000 persons [8]. Reduced corneal sensation, as seen in NK, can lead to recurrent and persistent corneal epithelial defects, ulcers, corneal opacification, and even perforation, posing a serious threat to vision in addition to negatively impacting the quality of life [1, 7, 9-13]. Patients are at greater risk of complications and vision loss if they have a concomitant facial nerve palsy with lagophthalmos, which can lead to chronic exposure keratopathy and dry eye [9-11]. However, when corneal sensation is severely reduced, breakdown of the corneal epithelium can occur even without provoking factors such as excess dryness, infection, exposure, or trauma. Furthermore, epithelial defects can persist without proper healing [14].

NK can be unilateral or bilateral and can result from any cause that leads to injury or impairment of the trigeminal nerve or one of its branches, specifically the ophthalmic branch [7]. Common causes include herpetic keratitis; systemic diseases such as diabetes mellitus, multiple sclerosis, and leprosy; chemical and thermal eye injury; long-term contact lens use; topical anesthetic abuse, injury to the trigeminal nerve secondary to an intracranial space-occupying lesion, such as an acoustic neuroma; nerve transection secondary to anterior segment surgery or refractive surgery; or neurosurgical procedures that damage the trigeminal ophthalmic branch $[4,5,7,15,16]$.

The Mackie classification helps to stage the severity of disease and outlines appropriate interventions. According to this classification system, stage 1 represents early disease with corneal epithelial hyperplasia, superficial punctate keratopathy, superficial neovascularization and scarring, and dellen formation. Stage 2 is moderate disease with persistent epithelial defects with smooth and rolled edges, Descemet's folds, and stromal swelling that can progress to anterior chamber inflammation with hypopyon. Stage 3 is severe disease, such as corneal ulcer with stromal stomal melt that progresses to perforation $[4,5,17 \cdot, 18,19]$. An estimated 1 in 10,000 persons suffer from severe (stage 3) NK [17•].

Conventional therapy for NK depends on the severity of disease and focuses primarily on protecting the ocular surface via lubrication (artificial tears, ointment), bandage contact lenses, amniotic membrane transplantation, or tarsorrhaphy. The field is rapidly changing, however, and in recent years, numerous new surgical and medical treatments have developed that aim to restore proper corneal innervation and promote ocular surface healing. We review the development of various corneal neurotization $(\mathrm{CN})$ techniques and discuss the advantages and disadvantages of each approach based on the donor nerve and nerve graft selections, degree of invasiveness, and clinical outcomes. We also present novel medical therapies that have emerged as alternatives to corneal neurotization in the treatment of moderate to severe NK.

\section{Corneal Neurotization}

$\mathrm{CN}$ is a rapidly evolving surgical procedure that involves the transfer of healthy donor sensory nerve axons to the denervated cornea in order to reestablish innervation. $\mathrm{CN}$ has shown success at restoring corneal sensation by reinnervating the stromal and sub-basal layers of the cornea, with a metareview of 54 eyes showing significant improvement in both best-corrected visual acuity (BCVA) and corneal sensation following neurotization $[20 \bullet, 21]$. This surgical approach has been demonstrated in both humans and animal models to effectively protect the cornea from ulceration and perforation and to promote faster epithelial healing compared to nonneurotized neurotrophic corneas [3, 5, 10, 11, 22-24]. Since CN was first introduced by Terzis et al. in 2009 [10], a series of adaptations and new techniques have evolved.

\section{Direct Nerve Transfer}

The direct method of $\mathrm{CN}$ involves directly transferring intact donor sensory nerves to the corneoscleral limbus of the anesthetic cornea. Various approaches and donor nerve selections have been described and are summarized here. 


\section{Coronal Approach for Direct Nerve Transfer}

Terzis et al. first described the coronal approach for $\mathrm{CN}$ as a method to directly transfer the contralateral supraorbital nerve (SON) and supratrochlear nerve (STN) to the anesthetic cornea [10]. This technique was modified by Jacinto et al. in 2016 [24], who described direct transfer of the ipsilateral SON for the same indication. The coronal approach involves elevating a large scalp flap that provides excellent exposure for atraumatic transfer of the SON and STN. These nerves exit the orbit anteriorly, providing sensation to portions of the forehead and scalp, and are considered good choices for donor nerves because they are large enough to be dissected under magnification from their original anatomic locations with reasonably low risk and are sufficient in length to reach their intended target [10].

The surgical approach begins with a bicoronal incision $3 \mathrm{~cm}$ behind the hairline from one auricular helix to the other, or from midline to the ipsilateral auricular helix (hemicoronal) in the case of ipsilateral donor nerve harvest. The flap is dissected in a subgaleal plane to the supraorbital rims for contralateral transfer (Fig. 1), or the ipsilateral orbital rim for ipsilateral transfer. The SON and STN are then dissected proximally to the supraorbital margin using high magnification. Approximately 4 to $10 \mathrm{~cm}$ of the nerve can be dissected, depending on the patient's anatomy and the distance to the ipsilateral versus contralateral cornea. The nerve branches are tunneled over the nasal bridge for contralateral transfer, or over the orbital rim for unilateral transfer, to a small incision along the upper eyelid crease of the affected eye. A blepharotomy is created between the superior fornix and the eyelid crease incision, medial to the medial horn of the levator aponeurosis, and the nerve branches are retrieved from the eyelid incision to the fornix using a hemostat. The distal nerve branches are then tunneled in a sub-Tenon's plane to an

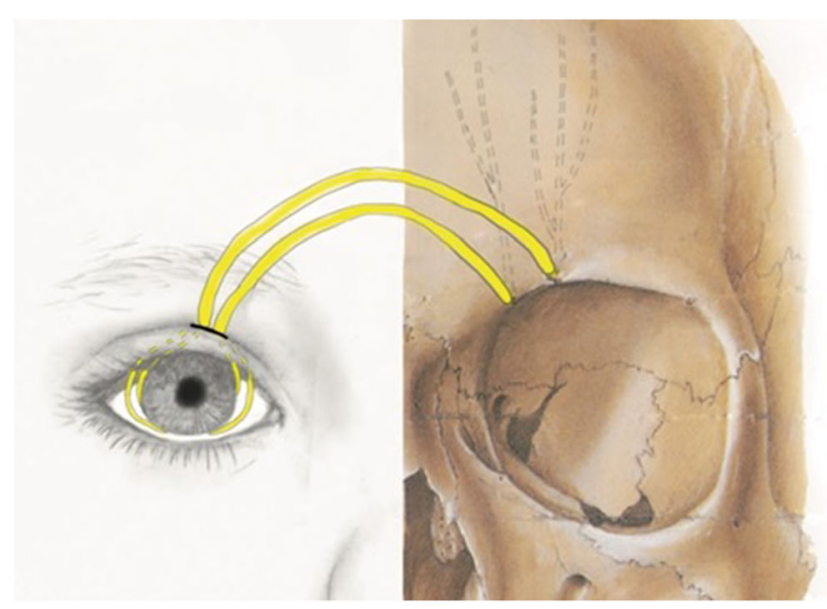

Fig. 1 Direct corneal neurotization. The contralateral supraorbital and supratrochlear branches are harvested and tunneled across the bridge of the nose and inserted around the limbus of the contralateral anesthetic eye. Figure from Terzis et al. Plast Reconstr Surg. Jan 2009 incision made $7 \mathrm{~mm}$ above the superior limbus to conjunctival incisions made at the 12,3,6, and 9 o'clock positions at the corneoscleral limbus. The donor nerve is inset by securing the distal nerve branches to the sclera within $1 \mathrm{~mm}$ of the corneoscleral limbus using 8-0 to $10-0$ absorbable or nonabsorbable sutures with or without fibrin glue [3, 10].

Terzis et al. reported improvement in corneal sensation, visual acuity due to resolution of corneal clouding, and corneal health with no ulcer recurrence or signs of advancing NK, at a mean follow-up of 16.3 months [10]. Advantages of the coronal approach include the readily accessible donor sensory nerves in areas of anatomic familiarity to most plastic surgeons without preparation of graft tissues, a single location of postoperative hypoesthesia, anesthesia, or potential scars, and avoidance of a neurorrhaphy that may increase the risk of neuroma formation and prevent successful nerve regeneration [10]. Disadvantages include possible insufficient donor nerve length to reach the contralateral cornea, increased risk for iatrogenic nerve injury from manipulation when harvesting long lengths of the nerve, and axonal loss at distal ends of the nerve that could limit the overall efficacy. Complications include hematoma or neuroma formation and potential numbness and discomfort at the donor site, although this typically resolves within a few months.

\section{Minimally Invasive Direct and Endoscopic Direct Nerve Transfer}

The evolution of $\mathrm{CN}$ has led to the development of minimally invasive direct corneal neurotization (MIDCN) approaches, such as the techniques described by Leyngold et al. In these techniques, endoscopic guidance, rather than a bicoronal or hemicoronal incision, can aid in contralateral SON transfer $[25,26]$. For unilateral direct nerve transfer, a single upper eyelid crease incision with direct visualization can be used to harvest the ipsilateral SON [27].

The MIDCN technique involves an upper eyelid crease incision below the donor supraorbital nerve for ipsilateral transfer. For contralateral nerve transfer, an additional contralateral eyelid crease incision is needed as well as two $1-\mathrm{cm}$ vertical scalp incisions, one midline and one tangent to the contralateral medial limbus, similar to an endoscopic brow lift. The donor neurovascular bundle is identified through the eyelid crease incision. Dissection is then continued superiorly along the subgaleal plane to isolate a $1-\mathrm{cm}$ segment of the periosteal branch of the SON. In ipsilateral transfer, approximately $4 \mathrm{~cm}$ of the SON is dissected under direct visualization (endoscopic guidance is not needed), the distal end is transected, and the nerve is reflected inferiorly through the ipsilateral upper eyelid incision. In contralateral transfer, an endoscope is used via the scalp incisions to aid visualization. Subperiosteal dissection is carried out until just cephalad to the isolated segment of SON. The periosteum is incised and the SON branches are isolated 
(Fig. 2). Approximately $8 \mathrm{~cm}$ of the nerve is dissected until its distal end is severed through a scalp incision. Similar to previously described methods, the nerve branches are then tunneled to the contralateral eyelid crease incision, to the fornix through a blepharotomy incision, and tunneled to the corneoscleral limbus in a sub-Tenon's plane.

Outcomes for the endoscopic technique have been reported for five patients with promising results including increased corneal sensation measured by Cochet-Bonnet (CB) esthesiometry and improved corneal appearance [28, 29]. The SON is the preferred donor nerve in MIDCN as it has consistent anatomy, reliable nerve length, and contains more myelinated axons than the STN. However, it should be noted that there are equally successful reports of corneal sensation return using the STN [30]. MIDCN for unilateral nerve transfer has demonstrated successful outcomes with improved or stable BCVA and rapid recovery of corneal sensibility at 24 months, measured with $\mathrm{CB}$ esthesiometry, without persistent epithelial defects [27].

Advantages of the endoscopic MIDCN approach include reduced morbidity, faster postoperative healing and surgical time, improved cosmesis, improved postoperative forehead sensation, and the ability to use either ipsilateral or contralateral SONs. Disadvantages include the cost of endoscopic instrumentation and the technique's learning curve, with moderate technical difficulty for operating surgeons [27]. Advantages of the MIDCN for unilateral nerve transfer include a localized area of incision and dissection that decreases the risk of postoperative morbidity or donor nerve injury, shorter operative times, and the flexibility to use general or intravenous anesthesia [27].

\section{Indirect Nerve Transfer}

The indirect method of $\mathrm{CN}$, also termed minimally invasive corneal neurotization (MICN), involves the use of an interpositional nerve graft that serves as a conduit between the donor sensory nerve and the anesthetic eye (Figure $3 a-d)[3,31$, 32]. Variations to this technique include the choice of the interpositional nerve graft, which includes several autograft and allograft options, as well as the donor nerve selection. Once selected and harvested, the end of the nerve graft is connected to the cut end of the donor sensory nerve (end-to-end) or to a cut epineurial window in the side of the donor nerve (end-toside) using sutures or fibrin adhesives. This connection is termed coaptation. The advantages of the indirect method include the ability to access the donor nerve proximally, where a greater number of axons are available for regeneration. Furthermore, minimally invasive eyelid or conjunctival incisions obviate the need for large coronal incisions and extensive facial dissections. Additional advantages include preservation of donor site sensation and surgical flexibility to choose nerve grafts and coaptation techniques to meet the anatomical constraints. Disadvantages include potential complications at the neurorrhaphy site such as neuroma formation [23, 27], and the increased distance for nerve regeneration, which may result in delayed corneal reinnervation as compared with the direct approach.

While the interpositional graft can be coapted to a variety of donor sensory nerves, Elbaz et al. were the first to report the coaptation of an interpositional graft to the ipsilateral or contralateral STN and SON [32]. The STN or the SON is accessed via a medial upper lid incision, just inferior to the brow. The end of the interpositional nerve graft is then coapted to either the side of the donor nerve (end-to-side) or to the end of the donor nerve (end-to-end). The advantage of end-to-side coaptation is that the donor sensory nerve is preserved, which lessens donor nerve site morbidity and loss of donor site sensation. Sutures, amniotic membrane, and/or fibrin glue may be used to reinforce the neurorrhaphy site. The distal nerve graft is then tunneled to the corneoscleral limbus in a similar manner as previously described.

\section{Autograft Options}

Autograft options include the sural nerve, greater auricular nerve, and lateral antebrachial cutaneous nerve. Autologous nerve grafts provide an optimal environment for axon regeneration and supply sufficient nerve length to allow for coaptation with a variety of contralateral and ipsilateral donor
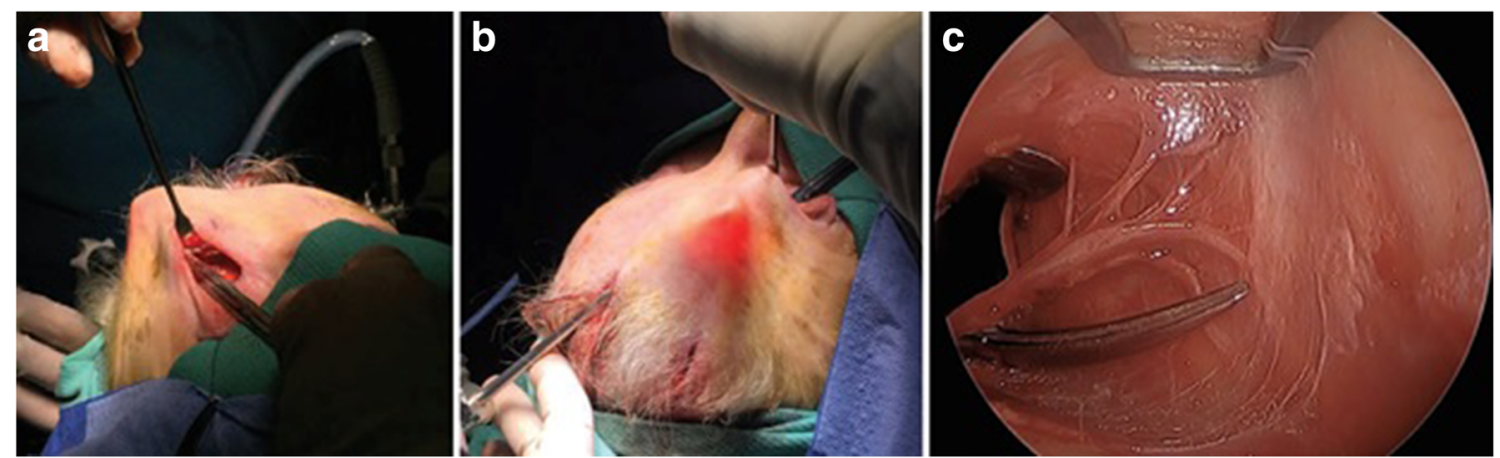

Fig. 2 Minimally invasive direct corneal neurotization. Endoscopic dissection of the supraorbital nerve from the eyelid (a) and scalp (b) incisions. Supraorbital nerve being harvested under endoscopic visualization (c). Figure from Leyngold et al. Ophthal Plast Reconstr Surg. Jan/Feb 2018 


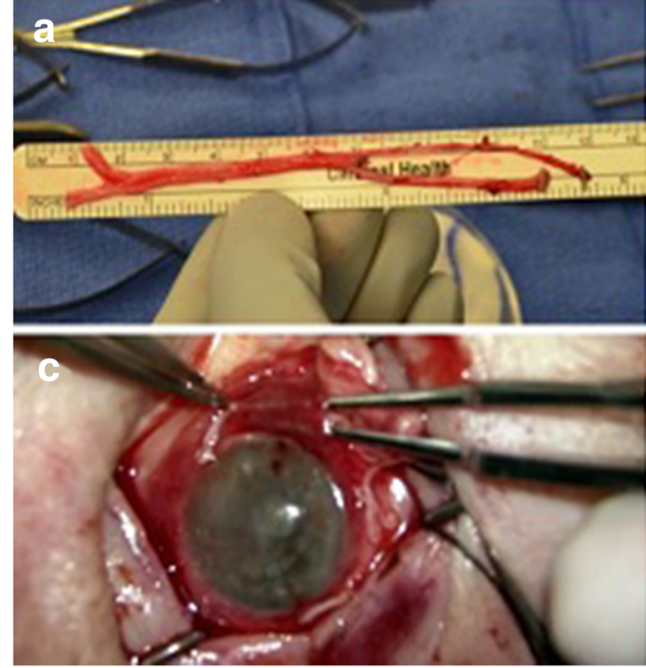

Fig. 3 Indirect corneal neurotization. a A segment of sural nerve is harvested. In this photo, two segments of the sural nerve are shown. b The distal end of the nerve graft is separated into fascicles on the ocular surface. c The fascicles are tunneled in a sub-Tenon's plane around the limbus to the desired quadrants and secured to the corneoscleral limbus. d

sensory nerves. However, disadvantages include graft site morbidity, such as numbness, scarring, and increased infection risk [32], and the need for a second team to harvest the graft, which may prolong operative times. Several interpositional graft options are described below.

\section{Sural Nerve Graft}

Elbaz et al. first described the use of the sural nerve (SN) for indirect CN in 2014, where a segment of the medial cutaneous branch of the $\mathrm{SN}$ was used as an interpositional graft between the STN donor nerve and the anesthetic cornea [32]. The medial cutaneous branch of the $\mathrm{SN}$ is harvested from the leg from the proximal to the distal end to obtain $10-15 \mathrm{~cm}$ of the nerve graft. This portion of the SN provides sensation to the distal lower leg and dorsum of the foot and can be harvested with minimal morbidity. A review of 19 eyes of young patients with mostly grade $2-3 \mathrm{NK}$ that underwent $\mathrm{CN}$ with this technique showed significant improvements in the number of episodes of epithelial defects and improved central corneal sensation by $\mathrm{CB}$ esthesiometry at a mean follow-up of 24 months [22]. Although the BCVA remained stable, immunochemical staining and magnetoencephalography confirmed the reinnervation of the cornea [22]. The SN graft has the ability to provide over $20 \mathrm{~cm}$ in length, which expands the donor nerve options to include the maxillary and mandibular branches of the trigeminal nerve [33]. Reports using the SN graft to the SON or STN demonstrate improvement in corneal healing, corneal sensation, and BCVA [5, 24, 33].

Jowett and Pineda reported end-to-end coaptation of the SN graft to the ipsilateral GAN, which has the benefit of being a large donor nerve with increased axon

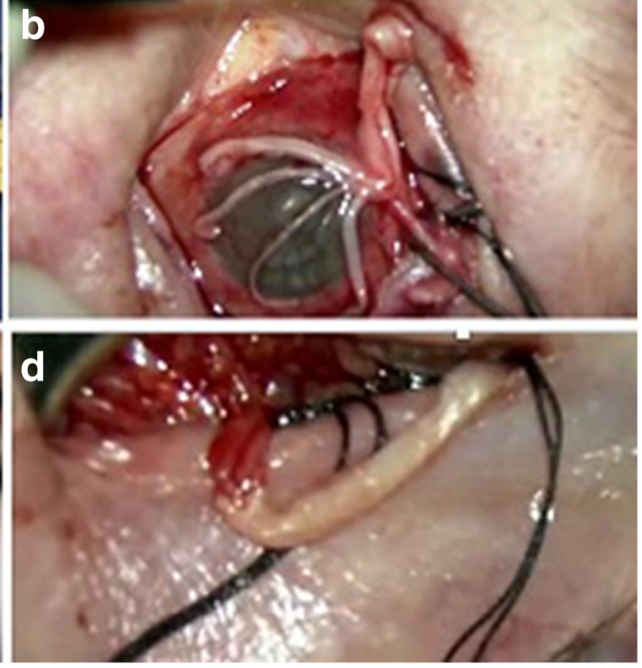

The proximal end of the nerve graft is coapted to the end of the supraorbital nerve using 10-0 nylon. A nerve wrap and fibrin glue can be added (not shown here). Alternatively, the nerve can be coapted endto-side or end-to-end to other donor nerves

count and results in reduced loss of facial sensation compared with the use of the SON and STN [34]. Disadvantages of this technique include a low likelihood of restoring a normal blink reflex and risk of injuring the interposition graft during any subsequent mid-facial surgeries [34].

\section{Greater Auricular Nerve Graft}

The greater auricular nerve (GAN) can also be used as an interpositional graft for $\mathrm{CN}$. It provides sensation to the skin over the parotid gland via its anterior branch and to the skin over the mastoid process, lower dorsal earlobe, and auricle via its posterior branch [34, 35]. Approximately $7 \mathrm{~cm}$ of the GAN can be harvested via a $3-\mathrm{cm}$ skin incision in the neck along the middle portion of the nerve and dissected towards the exit point of the GAN, which is about $6.5 \mathrm{~cm}$ from the mastoid and $1 \mathrm{~cm}$ from the external jugular vein [35]. This technique has demonstrated successful corneal nerve regeneration by confocal microscopy in multiple patients with improvement in BCVA and corneal sensation in the majority of patients $[34,35]$. The advantages of the GAN include use of a single surgical field to obtain the graft length needed to cover the interpupillary distance, a high number of myelinated axons serving as a good anatomical match with the STN, and limited area of postoperative anesthesia to the earlobe [35]. Use of a GAN graft, however, requires a challenging two-team approach due to limited space around the patient's head, which could increase the overall operating time [35]. 


\section{Antebrachial Cutaneous Nerve}

The antebrachial cutaneous nerve (LACN) is a purely sensory nerve that provides sensation to one-third of the anterior and posterior forearm and is easily accessed under the skin of the forearm, yielding a 12-cm nerve graft that includes the LACN and its terminal branches. Similar to the previously described techniques, the graft can be coapted to a donor nerve for indirect neurotization. A case report of $\mathrm{CN}$ using the $\mathrm{LACN}$ showed an improvement in corneal sensation and visual acuity [36]. The advantanges of the LACN graft include its long length with several terminal branches, an anatomical match with the SON, and transient loss of senation in a noncritical cutaneous region of the anterolateral forearm due to coinnervation by the radial sensory nerve. As with other indirect autologous grafts, a second team is needed for the graft harvest, which may lengthen surgical times [36].

\section{Acellular Nerve Allograft}

More recently, acellular nerve allografts (ANA) have gained popularity as they avoid the need for a second surgical team for graft harvesting and eliminate graft site morbidity. ANAs provide a commercially available nonimmunogenic scaffold to guide the regenerating donor nerve fibers to the target cornea. Leyngold et al. were the first to describe the use of ANAs, as opposed to a harvested nerve autograft, for $\mathrm{CN}$ [27]. ANAs can be coapted to the ipsilateral or contralateral SON, STN, or infraorbital nerve (ION). When using the SON or STN, the donor nerve is dissected and severed $1-2 \mathrm{~cm}$ distal to the orbital rim. When using the ION, the orbit is accessed via an inferior transconjunctival fornix incision, and a small portion of the orbital floor is removed over the infraorbital canal to access the ION. ANAs can be coapted to the donor nerve endto-end or end-to-side. In the case of end-to-end coaptation, a nerve connector or amniotic membrane graft can be used to protect the neurorrhaphy. In a report of seven patients across three hospital centers using ANAs coapted to the SON, STN, and ION, all patients demonstrated improved peripheral corneal sensation, with central corneal sensation improvement reported in 5 patients [27]. This technique provides the benefits of indirect $\mathrm{CN}$ without the added invasiveness and potential subsequent morbidity of harvesting a nerve autograft. Although it is thought that ANAs are devoid of viable Schwann cells that support axon regeneration and, therefore, they may have reduced growth support for regenerating axons compared with autografts, studies have shown reliable ANA axon regrowth over 3-4-cm distances [37, 38]. An observational study demonstrated no significant differences in outcome when stratified by nerve gap lengths of 10-24 mm, 25-49 $\mathrm{mm}$, and 50-70 $\mathrm{mm}$ [37]. However, the long-term efficacy in longer nerve allografts, as would be needed in contralateral neurotization, is still unknown. Furthermore, the cost and availability ANAs is a limitation of this technique. Nonetheless, early reports demonstrate return of corneal sensation when using the allograft coapted to the ipsilateral, infraorbital, supraorbital, supratrochlear, and even mobilized contralateral supraorbital or supratrochlear donor nerves [37-39].

\section{Additional Modifications}

In addition to variations in nerve selection and overall surgical approach, $\mathrm{CN}$ techniques may employ different methods for how donor nerves and grafts are joined at the neurorrhaphy site and how the nerve fascicles are inset at the corneoscleral limbus. With regard to the neurorrhaphy site, options include sutures, plus or minus fibrin glue adhesive, and with or without a protective membrane wrap. Sutures can be used to assure a secure connection, but this carries a small risk of nerve damage. Fibrin is quick and easy to apply, but if used alone it may weaken before nerve healing is complete, leading to nerve gaps and resulting failed neurotization [5, 13, 34]. Nerve wrap options include amniotic membrane, porcine extracellular matrix, and absorbable collagen implant, which can be applied with fibrin glue and has anti-inflammatory properties that can reduce adhesions and scarring at the neurorrhaphy site $[3,5,27]$. At the corneoscleral limbus, some surgeons secure the nerve fascicles to the perilimbal episcleral space using absorbable or non-absorbable $8-0$ to $10-0$ sutures $[25$, 35]. Other surgeons choose to secure the fascicles to the corneoscleral limbus using fibrin glue alone or a combination of the two [22]. Another option is to place the fascicles in perilimbal scleral tunnels, which bring the nerve fascicles into closer contact with the cornea but are technically challenging to execute and can lead to nerve fascicle damage and weaken the cornea's structure $[3,22,31,32]$. Studies have shown that the differences in $\mathrm{CB}$ esthesiometry between subconjunctival and corneoscleral tunnel placement of nerve fascicles disappear by 6 months with equivalent long-term results [40]. Further studies on the comparison of various modifications in neurorrhaphy and limbal attachment techniques are still needed.

\section{Medical Therapies}

While $\mathrm{CN}$ focuses on reinnervating the anesthetic cornea, conventional medical approaches to NK management have centered on promoting lubrication and protection of the ocular surface. In recent years, however, novel medical therapies have been developed to stimulate nerve growth and restore proper healing of the ocular surface. Several topical and oral medications provide potential non-invasive options for improving corneal nerve health and maintenance of the corneal epithelial surface. 


\section{Nerve Growth Factor}

Nerve growth factor (NGF) is a neurotrophic factor released by corneal nerves that helps with corneal repair and maintenance of homeostasis of the ocular surface [1]. It contributes to the growth, migration, and survival of neurons, promotes corneal epithelial cell proliferation, and stimulates corneal epithelial healing by increasing substance $\mathrm{P}$ and calcitonin generelated peptides [3, 7, 41]. In a rat model, NGF levels were shown to transiently increase in response to corneal epithelial injury, suggesting that NGF plays a role in the nerve injury response and regeneration of injured neurons [42-44]. Preliminary clinical studies showed efficacy and safety of murine NGF drops in promoting epithelial healing in moderate to severe NK $[45,46]$. To better suit further clinical development, drops using recombinant human NGF have been formulated and confirmed as safe in phase I trials [47]. Successful outcomes with regard to epithelial healing led to the approval of Cenegermin (Oxervate ${ }^{\mathrm{TM}}$ ) by the Food and Drug Administration (FDA) and European Medicines Agency (EMA) as a treatment for moderate to severe NK following phase II trials $[15,45]$.

\section{Cenegermin (Oxervate ${ }^{\mathrm{TM}}$ )}

Cenegermin, a recombinant human nerve growth factor (rhNGF), produced by Escherichia coli is the first medical therapy approved for the treatment of stage 2-3 NK. Two phase II randomized clinical trials have shown that rhNGF eye drops are safe and effective in the treatment of NK. Cenegermin was shown to improve corneal epithelial healing, defined as $<0.5 \mathrm{~mm}$ fluorescein staining, in up to 69.6-74.5\% of patients with moderate to severe NK at 8 weeks [15]. No residual epithelial staining was noted in $65.2 \%$ of patients in the US study at the 8-week follow-up, although 6 patients eventually had a recurrence of persistent epithelial defects. Of these six patients, 5 were retreated with a second round of 8 weeks of topical rhNGF drops, with 4 patients demonstrating healing with fluorescein stains $<0.5 \mathrm{~mm}$. Among patients that achieved corneal healing in a European study, 3.4$3.6 \%$ had a recurrence of epithelial defects at 48 weeks of follow-up $[15,17 \cdot]$. Notably, these studies failed to show a significant improvement in central corneal sensation or BCVA in patients receiving $20 \mu \mathrm{g} / \mathrm{ml}$, which is the FDA approved and currently available dosage in the market $[15,48 \bullet \bullet]$. Disadvantages include mild transient ocular pain, which has been attributed to the normal healing and the corneal nerve restorative process as well as the long treatment period of 8 weeks, frequent instillation of drops every $2 \mathrm{~h}$, and high cost.

Additional studies have shown that cenegermin is safe and effective for use in pediatric patients $\geq 2$ years old and is now permitted for use in children in the USA [49]. The most common side effects of cenegermin include eye and eyelid pain, inflammation, increased tearing, and foreign body sensation in the eye [50]. No current concensus on timing of cenegermin for the treatment of NK is established; however, it is generally reserved for treatment of moderate to severe NK to aid corneal healing and prevent progression to corneal melt. As of yet, there are no clinical guidelines on retreatment with cenegermin for NK that recurs or persists after 8 weeks of initial cenegermin treatment; however, successful resolution of epithelial defects was seen in four of five patients who were retreated within 1-104 days of the last cenegermin dose in the phase II trial [45].

\section{Nicergoline (Sermion ${ }^{\circledR}$ )}

Nicergoline is a derivative of ergoline that is typically used to treat cognitive impairment due to degenerative dementia or stroke [50,51]. Administered orally, the drug is thought to increase the healing of corneal epithelial wounds by increasing levels of NGF in the cornea and lacrimal glands, as evidenced by rat studies $[42,52]$. A prospective non-comparative study in 27 eyes of 24 patients from South Korea showed complete healing of corneal epithelial defects in $86 \%$ of treated eyes within an average of 15.6 days after twice-daily treatment with $10 \mathrm{mg}$ of oral nicergoline [42]. In addition, this study found significant improvement in central corneal sensitivity by $\mathrm{CB}$ esthesiometry with an improvement of $>10 \mathrm{~mm}$ in $52 \%$ of eyes, improvement in BCVA by $>1$ line of the Snellen test in $63 \%$ of eyes (>2 line improvement in 33\% of eyes), and increased tear NGF levels. Patients who demonstrated epithelial healing showed no evidence of recurrence at an average of 6.1 months of follow-up [17•, 42]. This study lacked a control group, and its small sample size limits the significance of the findings. Although nicergoline oral therapy shows promise in terms of promoting corneal epithelial healing, additional high-level evidence, such as a randomized control trial, is needed to support its use for the treatment of NK.

\section{Substance P/Insulin-Like Growth Factor}

Substance $\mathrm{P}$ is a neurotransmitter that is released from corneal nerve fibers in response to inflammation. The neuropeptide works in conjunction with insulin-like growth factor 1 (IGF1) and epidermal growth factor (EGF) to promote epithelial proliferation and corneal wound healing [1, 53, 54]. A formulated topical drug has been tested in an open-label study of 26 eyes with stage 1-2 NK in Japan [55]. The results demonstrated resolution of epithelial defects in up to $82 \%$ of patients at 10.5 days, improvement in visual acuity in $65 \%$ of study eyes but minimal corneal sensation return as measured by $\mathrm{CB}$ esthesiometry [55]. A separate case report also confirmed the regeneration of corneal nerve fibers through in vivo confocal microscopy [56]. However, there is no evidence to 
support the use of substance P/insulin-like growth factor (Sub$\mathrm{P} /$ IGF) in advanced NK. Thus, while early study results are encouraging, additional research is needed to further evaluate the efficacy of Sub-P/IGF eye drops across all stages of NK.

\section{Serum Tears}

Serum tears are a topical treatment made from a combination of autologous patient serum and blood derivatives, which have been shown to contain growth factors and neurotrophic factors such as substance P, IGF-1, NGF, EGF, and plateletderived growth factors that promote tissue regeneration and repair $[57,58]$. This treatment has been around for decades, used to relieve symptoms and promote re-epithelialization in a variety of ocular surface conditions including chemical burns and dry eye disease in patients with Sjögren's syndrome [59, 60]. Several retrospective serum tear studies of limited sample size have demonstrated an improvement in epithelial defects in early stages of NK with accompanying visual acuity improvement, and minimal to mild corneal sensation returns measured by $\mathrm{CB}$ esthesiometry and modified Belmonte esthesiometer [58, 61-64]. An observational study of 22 eyes of all stages of NK found favorable changes in the corneal nerves via the in vivo confocal microscopy, healing of the epithelial defect for $91 \%$ of patients within 12 weeks, and improvement in BCVA in $71 \%$ of patients [63]. The $9 \%$ of patients who did not demonstrate epithelial defect healing all had stage 3 NK. This suggests limited effectiveness in advanced NK, although other retrospective studies have demonstrated successful treatment of stage $3 \mathrm{NK}$ with autologous serum drops [58]. Higher level evidence is needed to confirm the efficacy of serum tears in the treatment of all stages of NK.

\section{Plasma Rich in Growth Factors (Endoret ${ }^{\oplus}$ )}

Plasma rich in growth factors (PRGF) is also an autologous blood-based therapy that has been used for NK. PRGF eye drops are comprised of platelet-enriched plasma from the patient's own blood that is activated using calcium chloride to release biologically active proteins, including antiinflammatory compounds and growth factors, such as EGF and NGF $[65,66]$. The compounds contained in the drops promote wound repair and help maintain the ocular surface, as demonstrated by proliferation and migration assay studies [66]. A retrospective study of 38 eyes by Sanchez-Avila et al. demonstrated complete resolution of corneal defect/ulcer in 97\% of eyes with stage $2-3$ NK treated with PRGF drops, as well as a statistically significant improvement in BCVA and a decrease in ocular symptoms such as discomfort, dryness, pain, foreign body sensation, burning/stinging, photophobia, and blurred vision. Corneal sensation was not tested in this study. Moreover, patients were treated with concomitant therapies as determined necessary by the clinician [67]. A phase-4 randomized clinical study of PRGF eye drops in patients with stages 2 and $3 \mathrm{NK}$ is ongoing (NCT02707120).

\section{Regenerating Agent (Cacicol ${ }^{\circledR}$ )}

Regenerating agents (RGTAs) are topical agents composed of polysaccharides in a variety of specific formulations that are made to mimic natural heparan sulfates, components of the corneal extracellular matrix [49]. The polysaccharides are linked to a matrix protein and growth factors and serve to restore the balance of matrix proteins and cytokines normally bound to heparan sulfate [67]. In doing this, RGTAs rejuvenate the stroma by providing binding sites for growth factors to promote healing and maintain homeostasis of the cornea's microenvironment. Furthermore, RGTA is suggested to prevent corneal epithelial degradation by mimicking the natural molecules that anchor and protect structural proteins and protect vital epithelial factors from destruction by proteolysis [49].

RGTA is currently commercially available in the European Union and the UK, and numerous studies have examined its safety and efficacy in treating a variety of ocular surface conditions $[17 \cdot, 68,69]$. A literature review of open-label studies and case series of RGTA as a treatment for NK showed complete corneal healing in $69 \%$ of eyes with a $4 \%$ recurrence rate; however, there was no improvement in corneal sensitivity [17•]. There is a growing consensus in favor of this agent in the treatment of stage $2 \mathrm{NK}$, although the results of a randomized double-blinded study evaluating RGTA20 efficacy versus placebo for neurotrophic and chronic corneal ulcers have yet to be released (NCT01242839) [49].

\section{Thymosin Beta 4}

Thymosin beta 4 (T $\beta 4)$ is a naturally occurring polypeptide, present in all tissues except red blood cells, that regulates the cellular actin cytoskeleton, cell migration, and inflammation. Treatment with $\mathrm{T} \beta 4$ promotes healing by combating the ocular inflammatory response and accelerates corneal epithelial growth by decreasing levels of cytokines such as TNF- $\alpha$, which trigger a signal cascade that activates numerous proinflammatory genes $[7,70,71]$. T $\beta 4$ promotes full-thickness wound healing, reduces inflammation by modulating infiltration of proinflammatory leukocytes and cytokines that cause swelling and tissue damage, and promotes endothelial and keratinocyte migration [7, 70-72].

Topical T $\beta 4$ was initially shown to be successful in animal models to treat rats with corneal epithelial injury and mice with ocular alkali burns [70, 71]. An in vitro study in human epithelial corneal cells stimulated with TNF- $\alpha$ showed significantly decreased activation of proinflammatory genes [71]. A case series of $\mathrm{T} \beta 4$ in nine eyes with stage $2 \mathrm{NK}$ and chronic nonhealing corneal epithelial defects demonstrated significant 
epithelial defect healing in $67 \%$ of patients, with complete defect healing in $44 \%$ of patients and no response in $33 \%$ of patients [73]. The beneficial role of T $\beta 4$ in the treatment of NK is yet to be determined, although a phase 3 randomized clinical study of RGN-259 ophthalmic solution for the treatment of NK (NCT02600429) is underway.

\section{Additional Medical Therapies}

Amniotic membrane extract eye drops (AMEED) aim to translate the known anti-inflammatory properties of amniotic membrane into an ophthalmic topical solution to promote corneal wound healing and regeneration in patients with NK [74, 75]. It may be used on its own or in a combined solution with umbilical cord blood and has been shown to successfully accelerate corneal epithelial healing in animal and human corneas [74, 76-79]. Isolated cases of successful resolution of epithelial defects with topical insulin and topical coenzyme Q10 in refractory cases of NK have been reported [80, 81]. The underlying mechanism remains unknown; however, topical insulin decreased the rate of sub-basal corneal plexus loss in diabetic mice and promoted epithelial cell migration in in vitro models $[82,83]$. Coenzyme Q10 (CoQ10) may promote epithelial healing by inhibiting cell apoptosis and reducing inflammation via NF-KB gene expression, and has been shown to improve the healing of chronic NK corneal ulcers when used as a topical solution formulated with vitamin $\mathrm{E}$ and hypromellose as an adjuvant therapy to conventional treatments [80]. Lifitegrast, an integrin antagonist that blocks LFA-1/ICAM-1 interaction to prevent $\mathrm{T}$ cell activation/ recruitment and further inflammation, has been FDAapproved for LASIK-related neurotrophic epitheliopathy [84]. Netrin-1, a class of proteins involved in axon guidance and cell migration, has been shown to promote epithelium repair by preventing apoptosis and facilitating cell proliferation and migration in mice studies and human corneal epithelial cells in vitro $[85,86]$. Further improvements in innovative medical therapies such as human-induced pluripotent stem cells have been the focus of interest in the treatment of corneal diseases and may show to improve outcomes in NK in the near future [87]. These emerging medical therapies still require rigorous study to better understand their mechanism, safety, and efficacy, but they provide promising new avenues for the expansion and improvement of primary and adjuvant treatments for NK.

\section{Discussion}

The field of NK management has changed drastically over the past decade, with numerous new surgical and medical treatments showing promising results. These methods aim to restore corneal innervation and/or promote the healing and maintenance of the ocular epithelial surface through a variety of pathways. As new treatment options become available, there are still unanswered questions regarding patient selection, when to start therapy, and which surgical or medical therapy is superior.

Proper patient selection is vital for successful surgical or medical therapy outcomes. While it is not clear which medical or surgical therapy is most appropriate for various stages of $\mathrm{NK}$, there are contraindications to $\mathrm{CN}$ which can guide medical therapy selection. Relative contraindications to $\mathrm{CN}$ include extensive conjunctival scarring, poorly controlled diabetics, patients with poor systemic health or overall poor surgical candidates, and anticoagulation use, and unrealistic patient expectations. Absolute contraindications include active or uncontrolled inflammatory or infectious ocular surface disease, perineural malignancy in the area of the donor nerve, absence of healthy sensory donor nerves, and ongoing external beam radiation to the orbit or eye [28]. In these patients, medical therapies should be considered.

The treatment of NK is also dependent on the stage of disease based on the Mackie classification. $\mathrm{CN}$ has been successfully performed for Mackie stage 1-3, while cenergemin is approved for Mackie stage 2-3 and RGTA has shown promising results in the treatment stage 2 NK $[10,15,22$, 49]. In the original $\mathrm{CN}$ study by Terzis et al., the Mackie stage 1 patients $(n=4,66.67 \%)$ had more return of corneal sensation compared to the stage 2 and 3 patients $(n=2,33.33 \%)$ [10]. Several authors advocate for early $\mathrm{CN}$ intervention in rapidly progressing NK to prevent permanent corneal scarring and avoid the need for penetrating keratoplasty or other ocular surface surgery [10]. Other authors, however, consider $\mathrm{CN}$ a procedure of last resort after medical therapies fail or reserve it for selected end-stage patients [88]. On the other hand, medical therapies such as rhNGF may be used in advanced NK in patients who are poor surgical candidates for $\mathrm{CN}$, or when the patient prefers a non-invasive approach. Currently, there are no established guidelines as to when patients should be offered treatment or which treatment to employ first. Specialists should discuss the risks and benefits of each option and customize therapy based on the patient's visual acuity, visual potential, Mackie stage, corneal sensation, underlying cause of NK, patient age, and systemic risk factors.

$\mathrm{CN}$ is a burgeoning field that restores innervation to the anesthetic cornea. Given the evolution in $\mathrm{CN}$ techniques, controversy exists over the best surgical approach. Various factors are important to consider prior to neurotization, including donor nerve selection, direct versus indirect approach, and interpositional nerve graft choice. A systematic review of all reported $\mathrm{CN}$ surgical outcomes demonstrated that neurotization leads to improved BCVA (logMAR improved to 0.85 from $1.25, p<.0001$ ) and central corneal sensation (CB esthesiometer improved from 2.18 to $40.10 \mathrm{~mm}$, $p<.0001$ ), with a median time of 8 months to maximal 
sensation return [20••]. Furthermore, a subgroup analysis by surgical techniques did not show significant differences in the clinical outcomes when controlled for age [20••].

There are several donor sensory nerve options, including the SON, STN, ION, and GAN, all with different distances, diameters, accessibility, and density of axons. Ideally, the donor nerve selected should be in closest proximity to the anesthetic cornea, with the highest number of axons, easily accessible, and carries minimal donor site morbidity. When performing indirect $\mathrm{CN}$, the donor and graft nerve diameters should match for optimal results. Generally, the number of myelinated axons is greater in the SON $(6017 \pm 4195)$ versus the STN $(2534 \pm 1667)$, while the GAN $(6530 \pm 2464)$ has a similar number of axons as the SON but is much further than the SON. The exact number of axons in the canal of the ION is unknown but is likely similar to the SON with a similar distance to the cornea; however, when the ION is utilized, it is typically through end-to-side coaptation, so it is unknown how many axons regenerate via this coaptation. Given the reliable postsurgical forehead sensory deficit recovery, the more proximal and larger SON is often the axon donor of first choice for many authors. However, the STN, ION, and GAN can be selected based on the reasons already mentioned, intact sensation and, surgeon preference.

In addition to donor nerve selection, the direct vs. indirect approach should be determined. Both have demonstrated successful outcomes despite certain surgical advantages and limitations. The MIDCN approach, using the ipsilateral SON donor, is an excellent option to address ipsilateral NK by keeping a short distance between the donor nerve and the affected cornea, and limiting the approach to one eyelid crease incision and one surgical team. When the contralateral SON or STN is selected as the donor, or in cases of bilateral corneal anesthesia, the direct nerve transfer approach is more challenging, and the indirect approach may be superior.

When selecting the indirect approach, surgeons should choose between an autologous nerve graft vs. an allograft. Controversy exists as to whether allografts are as effective as autologous nerve grafts for $\mathrm{CN}$. Allografts are attractive because they preserve autologous nerves, decrease surgical time, and come in a variety of diameters to prevent mismatch at the neurrorhaphy site. However, their lack of Schwann cells, which may decrease axon regeneration for longer lengths, suggest against its use for contralateral neurotization. Regardless of graft choice, the nerve graft diameter should be considered to select the best end-to-end diameter match. The end-to-end neurorrhaphy is the procedure of choice in the nerve surgery literature [89]; however, when the interpositional graft diameter is larger than the selected donor sensory nerve diameter, an end-to-side neurorrhaphy can be selected. A major advantage of end-to-side neurorrhaphy is that coaptation can be performed while preserving sensation in the distribution of the donor nerve. There are concerns of the amount of axon regeneration via end-to-side coaptation; however, the success of this technique is well documented [32]. Until more information is available to determine which donor nerve, approach, or graft option is superior, these surgical decisions should be tailored to the individual patient factors, outcome goals, surgeon preferences, and the availability of resources.

Novel medical treatments for NK provide an alternative yet effective and non-invasive option to $\mathrm{CN}$ (Figure 4). The topical rhNGF has been most rigorously studied via multiple randomized controlled trials and has been shown to improve corneal epithelial healing. Importantly, phase II randomized controlled trials showed no improvement in central corneal sensation or BCVA after 8 weeks of receiving $20 \mu \mathrm{g} / \mathrm{ml}$ of cenegermin $[15,45]$. Other studies on medical therapies remain observational and poorly controlled. The study characteristics are summarized in Table 1. Despite successful results in corneal epithelial healing, RGTA did not show any change in corneal sensation. Moreover, nicergoline and serum tears showed improved corneal healing and corneal sensation, although the improvement was minimal to mild (range 6$21 \mathrm{~mm}$ by CB esthesiometry) in mostly early stages of NK. This is in contrast to $\mathrm{CN}$ studies that showed significant improvement in both BCVA and central corneal sensation (average $38 \mathrm{~mm}$ by CB esthesiometry) [20••]. Furthermore, in vivo confocal microscopy and histological specimens of post-deep anterior lamellar keratoplasty following $\mathrm{CN}$ have also confirmed nerve growth in the treated corneas [21]. This suggests that $\mathrm{CN}$ and medical therapies improve the ocular surface environment in different ways. CN directly transfers nerves for nerve regeneration and long-term improvement in corneal health and sensation, while medical therapies provide the necessary trophic factors for epithelial regeneration (rhNGF, RGTA, Sub-P/IGF, serum tears, nicergoline) and decrease the overall inflammation (PRGF, RGTA) [49].

Disadvantages of $\mathrm{CN}$ include the invasiveness of the procedure, the time it takes to reestablish corneal sensation-a median 8 months to maximal sensation - and potential complications to surgery. For these reasons, rhNGF should be considered as a viable treatment option for moderate to severe $\mathrm{NK}$, especially in patients who are poor surgical candidates, where $\mathrm{CN}$ is contraindicated, or when immediate corneal healing is needed. An exciting area of research includes evaluating the potential synergistic effects of $\mathrm{CN}$ and rhNGF. Growth factors, including NGF, have been applied in nerve conduits to small animal models of nerve gap injury (1-4-cm gap) and demonstrate improved histological, electrophysiological, and functional outcomes compared with conduit controls [90]. It is possible that novel medical therapies could complement $\mathrm{CN}$, by providing the necessary trophic factors for more immediate corneal healing and by possibly enhancing axonal regeneration in $\mathrm{CN}$. Further studies are needed to compare $\mathrm{CN}$ and medical treatments, such as rhNGF, and to better understand the potential benefits of combined therapy. 


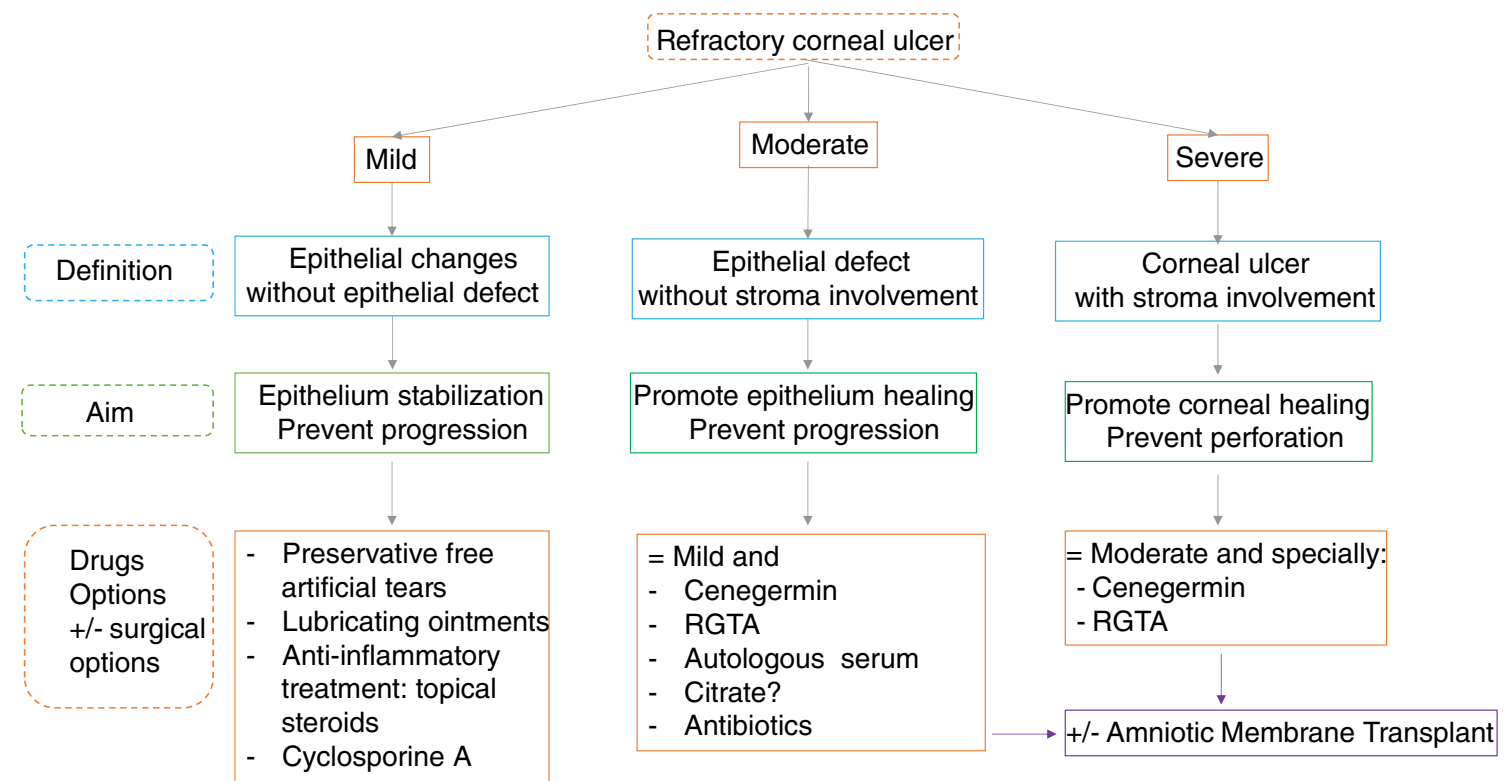

Fig. 4 Treatment approach for medical management of refractory corneal ulcers due to neurotrophic keratopathy (NK). Figure from Bremond-Gignac et al. Expert Opin Investig Drugs. Nov 2019

\section{Conclusion}

Numerous minimally invasive $\mathrm{CN}$ surgical techniques and novel medical therapies have resulted in a paradigm shift in
NK treatment. These new methods show promising results in their ability to resensitize the cornea, promote healing of the corneal epithelial surface, and restore the vital homeostasis between corneal nerves and the ocular surface.

Table 1 Summary of studies of medical therapies for neurotrophic keratopathy

\begin{tabular}{|c|c|c|c|c|c|c|c|c|}
\hline Study & Date & Treatment & $\begin{array}{l}\text { Number } \\
\text { of eyes }\end{array}$ & Stage & Design & $\begin{array}{l}\text { Follow-up period } \\
\text { (weeks) }\end{array}$ & $\begin{array}{l}\text { Improved } \\
\text { corneal } \\
\text { sensation }\end{array}$ & $\begin{array}{l}\text { Improved } \\
\text { BCVA }\end{array}$ \\
\hline Bonini et al. [47] & 2018 & rhNGF & 18 & $2-3$ & RCT & $48-56$ & Yes & Yes \\
\hline Bonini et al. [15] & 2018 & rhNGF & 156 & $2-3$ & RCT & $48-56$ & Yes & Yes \\
\hline Pedrotti et al. [50] & 2019 & rhNGF & 1 & 3 & Single case & 3 & Yes & No \\
\hline Sanchez-Avila et al. [67] & 2017 & PRGF-Endoret & 38 & $2-3$ & Case series & mean 11.4 (SD 13.7) & Yes & Yes \\
\hline Dunn et al. [73] & 2010 & $\mathrm{~T} \beta 4$ & 9 & 2 & Case series & 4 & - & - \\
\hline Yoon et al. [91] & 2007 & UCS & 28 & $1-3$ & Prospective open & $8-78$ & Yes & Yes \\
\hline Jeng et al. [61] & 2009 & AS $50 \%$ & 25 & 2 & Retrospective & 18 & - & - \\
\hline Matsumoto et al. [58] & 2004 & AS $20 \%$ & 14 & $1-3$ & Case series & $16-156$ & Yes & Yes \\
\hline Turkoglu et al. [62] & 2014 & AS $20 \%$ & 20 & $2-3$ & Retrospective & mean 26.8 (SD 8.2) & - & Yes \\
\hline Guadilla et al. [63] & 2013 & AS $20 \%$ & 22 & $1-2$ & Retrospective & 16 & Yes & Yes \\
\hline Rao et al. [64] & 2010 & AS & 11 & $1-2$ & Prospective open & $12-24$ & Yes & Yes \\
\hline Yanai et al. [92] & 2015 & IGF/Sub-P & 26 & $1-2$ & Prospective open & 12 & - & Yes \\
\hline Yamada et al. [55] & 2008 & IGF/Sub-P & 26 & 2 & Prospective open & 4 & Yes & Yes \\
\hline Nishida et al. [93] & 2007 & IGF/Sub-P & 11 & $1-2$ & Prospective open & 4 & - & Yes \\
\hline Guerra et al. [69] & 2017 & RGTA & 25 & 2 & Prospective not controlled & 12 & - & Yes \\
\hline Aifa et al. [94] & 2012 & RGTA & 11 & 2 & Prospective not controlled & $4-22$ & - & No \\
\hline Arvola et al. [95] & 2016 & RGTA & 8 & 2 & Case series & $6-10$ & - & No \\
\hline Lee et al. [42] & 2015 & Nicergoline & 27 & $2-3$ & Prospective controlled & 4 & Yes & Yes \\
\hline Gumus [80] & 2017 & CoQ10 & 6 & $1-3$ & Case series & 8 & - & Yes \\
\hline
\end{tabular}

$A S$ autologous serum, CoQ10 coenzyme Q10, IGF/Sub-P insulin-like growth factor/substance $\mathrm{P}, P R G F$ plasma rich in growth factors, $r h N G F$ recombinant human nerve growth factor, $R C T$ randomized controlled trial, $R G T A$ regenerating agent, UCS umbilical cord serum 
There are advantages and disadvantages to each $\mathrm{CN}$ technique, although all approaches have demonstrated to be effective at reinnervating the anesthetic cornea [20•0]. Additionally, medical therapies provide a non-invasive approach to the treatment of NK. Several new treatments show promising results such as rhNGF, RGTA, and nicergoline. However, not all medical treatments address the underlying problem in NK; therefore, it remains to be seen which options best facilitate long-term corneal health. Furthermore, many of these medical treatments are still lacking in higher level of evidence, such as serum tears, T $\beta 4$, PRGF, and Sub-P/IGF, to support their use for NK. Ultimately, the evolution of $\mathrm{CN}$ has led to the development of numerous successful methods for surgical corneal reinnervation, and several exciting new medical therapies provide viable non-surgical alternatives. Further research is needed to better understand the efficacy and effectiveness of these techniques and treatments, and how they can best be used alone or in combination to treat NK.

\section{Compliance with Ethical Standards}

Conflict of Interest The authors declare that they have no potential conflicts of interest.

Human and Animal Rights and Informed Consent All human and animal studies have been approved by the appropriate ethics committee and have therefore been performed in accordance with the ethical standards laid down in the 1964 Declaration of Helsinki and its later amendments. This manuscript does not contain any identifiable patient information.

Open Access This article is licensed under a Creative Commons Attribution 4.0 International License, which permits use, sharing, adaptation, distribution and reproduction in any medium or format, as long as you give appropriate credit to the original author(s) and the source, provide a link to the Creative Commons licence, and indicate if changes were made. The images or other third party material in this article are included in the article's Creative Commons licence, unless indicated otherwise in a credit line to the material. If material is not included in the article's Creative Commons licence and your intended use is not permitted by statutory regulation or exceeds the permitted use, you will need to obtain permission directly from the copyright holder. To view a copy of this licence, visit http://creativecommons.org/licenses/by/4.0/.

\section{References}

Papers of particular interest, published recently, have been highlighted as:

- Of importance

•- Of major importance

1. Yang AY, Chow J, Liu J. Corneal innervation and sensation : the eye and beyond. Yale J Biol Med. 2018;91(1):13-21.

2. Shaheen B, Bakir M, Jain S. Corneal nerves in health and disease. Surv Ophthalmol. 2014;59(3):263-85.

3. Wolkow N, Habib LA, Yoon MK, Freitag SK. Corneal Neurotization : review of a new surgical approach and its developments corneal neurotization : review of a new surgical approach and its developments. Semin Ophthalmol. 2019;34:1-15. https://doi.org/10.1080/08820538.2019.1648692.

4. Ting DSJ, Figueiredo GS, Henein C, Barnes E, Ahmed O, Mudhar HS, et al. Corneal neurotization for neurotrophic keratopathy : clinical outcomes and in vivo confocal microscopic and histopathological findings. Cornea. 2018;37(5):641-6.

5. Malhotra R, Sha M, Kannan R, Nduka C, Hamada S. Update on corneal neurotisation. Br J Ophthalmol. 2019;103(1):26-35.

6. Al-aqaba MA, Fares U, Suleman H, Lowe J, Dua HS. Architecture and distribution of human corneal nerves. Br J Ophthalmol. 2009;94(6):784-9. https://doi.org/10.1136/bjo.2009.173799.

7. Sosne G, Rimmer D, Kleinman HK, Ousler G. Thymosin Beta 4 : A potential novel therapy for neurotrophic keratopathy, dry eye, and ocular surface diseases. Vitam Horm. 2016;102:277-306.

8. Mastropasqua L, Dua HS, Calienno R. In vivo microscopic and optical coherence tomography classification of neurotrophic keratopathy. J Cell Physiol. 2019;234(5):6108-15. https://doi.org/10. $1002 /$ jcp. 27345 .

9. Allevi F, Fogagnolo P, Rossetti L, Biglioli F. Eyelid reanimation, neurotisation, and transplantation of the cornea in a patient with facial palsy. BMJ Case Rep. 2014. https://doi.org/10.1136/bcr2014-205372.

10. Terzis J, Dryer M, Bodner B. Corneal neurotization: a novel solution to neurotrophic keratopathy. Plast Reconstr Surg. 2009;123(1): $112-20$.

11. Terzis JK, Dryer MM, Bodner BI. Corneal Neurotization : a novel technique for the anesthetic cornea. Cornea. 2010;29(7):812-9.

12. Goins K. New insights into the diagnosis and treatment of neurotrophic keratopathy. Ocul Surf. 2005;3(2):96-110.

13. Dua HS, Said DG, Messmer EM, Rolando M, Benitez-del-Castillo JM, Hossain PN, et al. Neurotrophic keratopathy. Prog Retin Eye Res. 2018;66:107-31.

14. Pushker N, Dada T, Vajpayee R, Gupta V, Aggrawal T, Titiyal J. Neurotrophic keratopathy. CLAO J. 2001;27(2):100-7.

15. Bonini S, Lambiase A, Rama P, Sinigaglia F, Allegretti M, Chao W, et al. Phase II randomized, double-masked, vehicle-controlled trial of recombinant human nerve growth factor for neurotrophic keratitis. Ophthalmology. 2018;125(9):1332-43.

16. Allen VD, Malinovsky V. Management of neurotrophic keratopathy. Contact Lens Anterior Eye. 2003;26(3):161-5. https://doi.org/ 10.1016/S1367-0484(03)00027-4.

17. Di Zazzo A, Coassin M, Varacalli G, Galvagno E, De VA, Bonini S. Neurotrophic keratopathy : pros and cons of current treatments. Ocul Surf. 2019; (August). This study compares and contrasts the clinical outcomes of currently available medical therapies in the treatment of neurotropic keratopathy.

18. Hsu HY, Modi D. Etiologies, quantitative hypoesthesia, and clinical outcomes of neurotrophic keratopathy. Eye Contact Lens Sci Clin Pract. 2015;41(5):314-7. https://doi.org/10.1097/ICL. 0000000000000133.

19. Sacchetti M, Lambiase A. Diagnosis and management of neurotrophic keratitis. Clin Ophthalmol. 2014;8:571.

20.• Park J, Charlson E, Leyngold I, Kossler A. Corneal Neurotization: a review of pathophysiology and outcomes. Ophthalmic Plast Reconstr Surg. 2020; This paper is the most extensive systematic review of corneal neurotization outcomes in current literature and provides a meta-analysis of corneal sensation and visual acuity changes before and after.

21. Fung S, Catapano J, Elbaz U, Zuker R, Borschel G, Ali A. In vivo confocal microscopy reveals corneal reinnervation after treatment of neurotrophic keratopathy with corneal neurotization. Cornea. 2018;37(1):109-12.

22. Catapano J, Fung SSM, Halliday W, Jobst C, Cheyne D, Ho ES, et al. Treatment of neurotrophic keratopathy with minimally invasive corneal neurotisation : long-term clinical outcomes and 
evidence of corneal reinnervation. Br J Ophthalmol. 2019;103(12): 1724-31. https://doi.org/10.1136/bjophthalmol-2018-313042.

23. Jacinto F, Espana E, Padilla M, Ahmad A, Leyngold I. Ipsilateral supraorbital nerve transfer in a case of recalcitrant neurotrophic keratopathy with an intact ipsilateral frontal nerve : a novel surgical technique. Am J Ophthalmol Case Rep. 2016;4:14-7.

24. Sepehripour S, Lloyd MS, Plast F, et al. Surrogate outcome measures for corneal neurotization in infants and children. J Craniofac Surg. 2017;28(5):1167-70. https://doi.org/10.1097/SCS. 0000000000003677.

25. Leyngold I, Weller C, Leyngold M, Espana E, Black KD, Hall KL, et al. Endoscopic corneal neurotization : cadaver feasibility study. OPRS. 2018;34(3):213-6. https://doi.org/10.1097/IOP. 0000000000000913

26. Leyngold I, Weller C, Leyngold M, Tabor M. Endoscopic corneal neurotization : technique and initial experience. OPRS. 2018;34(1): 82-5. https://doi.org/10.1097/IOP.0000000000001023.

27. Leyngold IM, Yen MT, Tian J, Leyngold MM, Vora GK, Weller C. Minimally invasive corneal neurotization with acellular nerve allograft : surgical technique and clinical outcomes. OPRS. 2019;35(2): 133-40. https://doi.org/10.1097/IOP.0000000000001181.

28. Wisely CE, Rafailov L, Cypen S, Proia AD, Boehlke CS, Leyngold IM. Clinical and morphologic outcomes of minimally invasive direct corneal neurotization. OPRS. 2020;36(5):451-7.

29. Kim JS, Rafailov L, Leyngold IM. Corneal Neurotization for Postherpetic Neurotrophic Keratopathy: Initial Experience and Clinical Outcomes. OPRS. 2020. https://doi.org/10.1097/iop .0000000000001676 .

30. Lin C, Lai L. Herpetic corneal keratopathy management using ipsilateral supratrochlear nerve transfer for corneal neurotization. Ann Plast Surg. 2019;83(5):553-7.

31. Bains R, Elbaz U, Zuker R, Ali A, Borschel GH. Corneal neurotization from the supratrochlear nerve with Sural nerve grafts: a minimally invasive approach. Plast Reconstr Surg. 2015;135(2): 397-400. https://doi.org/10.1097/PRS.0000000000000994.

32. Elbaz U, Bains R, Zuker R, Borschel G, Ali A. Restoration of corneal sensation with regional nerve transfers and nerve grafts. JAMA Ophthalmol. 2014;132(11):1289-95.

33. Weis E, Rubinov A, Al-ghoul AR, Yau FM. Sural nerve graft for neurotrophic keratitis : early results. Can J Ophthalmol. 2018;53(1): 23-8.

34. Jowett N, Pineda R II. Corneal neurotisation by great auricular nerve transfer and scleral-corneal tunnel incisions for neurotrophic keratopathy. Br J Ophthalmol. 2019;103(9):1235-8. https://doi.org/ 10.1136/bjophthalmol-2018-312563.

35. Benkhatar H, Levy O, Goemaere I, Borderie V, Laroche L, Bouheraoua N. Corneal Neurotization with a great auricular nerve graft : effective reinnervation demonstrated by in vivo confocal microscopy. Cornea. 2018;37(5):647-50.

36. Bourcier T, Henrat C, Heitz A, Kremer S, Labetoulle M, Liverneaux P. Lateral antebrachial cutaneous nerve as autologous graft for mini-invasive corneal neurotization (MICORNE). Cornea. 2019;38(8):1029-32.

37. Safa B, Shores JT, Ingari JV, Weber RV, Cho M, Zoldos J, et al. Recovery of motor function after mixed and motor nerve repair with processed nerve allograft. Plast Reconstr Surg Glob Open. 2019;7(3):e2163. https://doi.org/10.1097/GOX. 0000000000002163

38. Salomon D, Miloro M, Kolokythas A. Outcomes of immediate allograft reconstruction of long-span defects of the inferior alveolar nerve. J Oral Maxillofac Surg. 2016;74:2507-14.
39. Brooks D, Weber R, Chao J. Processed nerve allografts for peripheral nerve reconstruction: a multicenter study of utilization and outcomes in sensory, mixed, and motor nerve reconstruction. Microsurgery. 2012;32:1-14.

40. Catapano J, Demsey DR, Ho ES, Zuker RM, Borschel GH. Crossface nerve grafting with Infraorbital nerve pathway protection: anatomic and histomorphometric feasibility study. Plast Reconstr Surg Glob Open. 2016;4(9):e1037. https://doi.org/10.1097/GOX .0000000000001037

41. Ferrari G, Chauhan SK, Ueno H, Nallasamy N, Gandolfi S, Borges $\mathrm{L}$, et al. A novel mouse model for neurotrophic keratopathy : trigeminal nerve stereotactic electrolysis through the brain. Invest Ophthalmol Vis Sci. 2011;52(5):2532-9. https://doi.org/10.1167/ iovs. 10-5688

42. Lee Y, Kim S. Treatment of neurotrophic keratopathy with nicergoline. Cornea. 2015;34(3):303-7.

43. Lambiase A, Rama P, Aloe L, Bonini S. Management of neurotrophic keratopathy. Curr Opin Ophthalmol. 1999;10(4):270-6.

44. Lambiase A, Manni L, Bonini S, Rama P, Micera A, Aloe L. Nerve growth factor promotes corneal healing: structural, biochemical, and molecular analyses of rat and human corneas. Invest Ophthalmol Vis Sci. 2000;41(5):1063-9.

45. Bonini S, Lambiase A, Rama P, Caprioglio G, Aloe L. Topical treatment with nerve growth factor for neurotrophic keratitis. Ophthalmology. 2000;107(7):1347-51.

46. Lambiase A, Rama P, Bonini S, Caprioglio G, Aloe L. Topical treatment with nerve growth factor for corneal neurotrophic ulcers. N Engl J Med. 1998;338(17):1174-80.

47. Bonini S, Lambiase A, Rama P, Filatori I, Allegretti M, Chao W, et al. Phase I trial of recombinant human nerve growth factor for neurotrophic keratitis. Ophthalmology. 2018;125(9):1468-71.

48.• Pflugfelder S, Massaro-Giordano M, Perez V, et al. Topical recombinant human nerve growth factor (cenegermin) for neurotrophic keratopathy: a multicenter randomized vehicle-controlled pivotal trial. Ophthalmology. 2020;127(1):14-26. This study outlines the results of a phase II clinical trial evaluating the efficacy of recombinant human nerve growth factor (rhNGF) for treatment of neurotrophic keratopathy and is one of the only published randomized controlled trials assessing one of the novel medical therapies for NK.

49. Bremond-Gignac D, Daruich A, Robert MP, Daruich A, Robert MP. Recent innovations with drugs in clinical trials for neurotrophic keratitis and refractory corneal ulcers. Expert Opin Investig Drugs. 2019;28(11):1013-20. https://doi.org/10.1080/13543784. 2019.1677605.

50. Pedrotti E, Bonetto J, Cozzini T, Fasolo A, Marchini G. Cenegermin in pediatric neurotrophic keratopathy. Cornea. 2019;38(11):1450-2.

51. Ogawa N, Asanuma M, Hirata H, Kondo Y, Kawada Y, Mori A. Cholinergic deficits in aged rat brain are corrected with nicergoline. Arch Gerontol Geriatr. 1993;16(2):103-10.

52. Giardino L, Giuliani A, Battaglia A, Carfagna N, Aloe L, Calza L. Neuroprotection and aging of the cholinergic system: a role for the ergoline derivative nicergoline (sermion). Neuroscience. 2002;109(3):487-97.

53. Kim S, Choi J, Joo C. Effects of nicergoline on corneal epithelial wound healing in rat eyes. Invest Ophthalmol Vis Sci. 2009;50(2): 621-5. https://doi.org/10.1167/iovs.08-2037.

54. Belmonte C, Acosta MC, Gallar J. Neural basis of sensation in intact and injured corneas. Exp Eye Res. 2004;78(3):513-25. https://doi.org/10.1016/j.exer.2003.09.023. 
55. Yamada N, Matsuda R, Morishige N, Yanai R. Open clinical study of eye-drops containing tetrapeptides derived from substance $\mathrm{P}$ and insulin-like growth factor-1 for treatment of persistent corneal epithelial defects associated with neurotrophic keratopathy. Br J Ophthalmol. 2008;92(7):896-900.

56. Morishige N, Komatsubara T, Chikama T, Nishida T. Direct observation of corneal nerve fibres in neurotrophic keratopathy by confocal biomicroscopy. Lancet. 1999;354(9190):1613-4.

57. Nakamura M, Nishida T, Ofuji K, Reid T, Mannis M, Murphy C. Synergistic effect of substance $\mathrm{P}$ with epidermal growth factor on epithelial migration in rabbit cornea. Exp Eye Res. 1997;65(3): 321-9.

58. Matsumoto Y, Dogru M, Goto E, Ohashi Y, Kojima T, Ishida R, et al. Autologous serum application in the treatment of neurotrophic keratopathy. Ophthalmology. 2004;111(6):1115-20. https://doi. org/10.1016/j.ophtha.2003.10.019.

59. Franchini M, Curciani M, Mengoli C, et al. Serum eye drops for the treatment of ocular surface diseases: a systematic review and metaanalysis. Blood Transfus. 2019;17(3):200-9.

60. Tsubota K, Goto E, Fujita H, Ono M, Inoue H, Saito I. Treatment of dry eye by autologous serum application in Sjögren 's syndrome. Br J Ophthalmol. 1999;83(4):390-5.

61. Jeng B, Dupps W Jr. Autologous serum 50\% eyedrops in the treatment of persistent corneal epithelial defects. Cornea. 2009;28(10): 1104-8.

62. Turkoglu E, Celik E, Alagoz G. A comparison of the efficacy of autologous serum eye drops with amniotic membrane transplantation in neurotrophic keratitis. Semin Ophthalmol. 2014;29(3):11926.

63. Guadilla A, Balado P, Baeza A, Merino M. Effectiveness of topical autologous serum treatment in neurotrophic keratopathy. Arch Soc Esp Oftalmol. 2013;88(8):302-6.

64. Rao K, Leveque C, Pflugfelder S. Corneal nerve regeneration in neurotrophic keratopathy following autologous plasma therapy. $\mathrm{Br}$ J Ophthalmol. 2010;94(5):584-91.

65. Sharma N, Goel M, Velpandian T, Titiyal JS, Tandon R, Vajpayee RB. Evaluation of umbilical cord serum therapy in acute ocular chemical burns. Invest Ophthalmol Vis Sci. 2011;52(2):1087-92. https://doi.org/10.1167/iovs.09-4170.

66. Anitua E, Sanchez M, Merayo-lloves J, De Fuente M, Muruzabal F, Orive G. Plasma rich in growth factors ( PRGF-Endoret) stimulates proliferation and migration of primary and reverts TGF- 1 - induced myodifferentiation. Invest Ophthalmol Vis Sci. 2011;52(9): 6066-73. https://doi.org/10.1167/iovs.11-7302.

67. Sanchez-Avila R, Merayo-Lloves J, Riestra A, et al. Treatment of patients with neurotrophic keratitis stages 2 and 3 with plasma rich in growth factors ( PRGF-Endoret ) eye-drops. Int Ophthalmol. 2018;38(3):1193-204.

68. Chappelet M, Chiquet C, Aptel F. Effect of a new matrix therapy agent in persistent epithelial defects after bacterial keratitis treated. Cornea. 2017;36(9):1061-8.

69. Guerra M, Marques S, Gil Q, et al. Neurotrophic Keratopathy: therapeutic approach using a novel matrix regenerating agent. J Ocul Pharmacol Ther. 2017;33(9):662-70. https://doi.org/10. 1089/jop.2017.0010.

70. Sosne G, Qiu P, Christopherson P, Wheater M. Thymosin beta 4 promotes corneal wound healing and modulates inflammatory mediators in vivo. Exp Eye Res. 2001;72:605-8. https://doi.org/10. 1006/exer.2000.0985.

71. Sosne G, Szliter E, Barret R, Kernacki K, Kleinman H, Hazlett LD. Thymosin beta 4 promotes corneal wound healing and decreases inflammation in vivo following alkali injury. Exp Eye Res. 2002;74:293-9. https://doi.org/10.1006/exer.2001.1125.
72. Sosne G, Qiu P, Christopherson PL, Kurpakus M. Thymosin beta 4 suppression of corneal NF k B : a potential anti-inflammatory pathway. Exp Eye Res. 2007;84(4):663-9. https://doi.org/10.1016/j. exer.2006.12.004

73. Dunn SP, Heidemann DG, Chow CYC, Crockford D, Turjman N, Angel J, et al. Treatment of chronic nonhealing neurotrophic corneal epithelial defects with thymosin $\beta$ 4. Ann N Y Acad Sci. 2010;1194(1):199-206. https://doi.org/10.1111/j.1749-6632.2010. 05471.x.

74. Murri M, Moshirfar M, Birdsong O, Ronquillo Y, Ding Y, Hoopes P. Amniotic membrane extract and eye drops: a review of literature and clinical application. Clin Ophthalmol. 2018;12:1105-12.

75. He H, Chen S, Chen Y, Hayashida Y, Zhu Y, Tseng S. Suppression of activation and induction of apoptosis in RAW264.7 cells by amniotic membrane extract. Invest Ophthalmol Vis Sci. 2008;49(10):4468-75.

76. Shahriari H, Tokhmehchi F, Reza M, Hashemi N. Comparison of the effect of amniotic membrane suspension and autologous serum on alkaline corneal epithelial wound healing in the rabbit model. Cornea. 2008;27(10):1148-50.

77. Liang L, Li W, Ling S, Sheha H, Qiu W, Li C, et al. Amniotic membrane extraction solution for ocular chemical burns. Clin Exp Ophthalmol. 2009;37(9):855-63.

78. Sheha H, Lian L, Hashem H, Ramzy M, ZaKi H. Amniotic membrane extract for acute ocular chemical burns. Tech Ophthalmol. 2010;8(4):146-50.

79. Dudok D, Nagdee I, Cheung K, et al. Effects of amniotic membrane extract on primary human corneal epithelial and limbal cells. Clin Exp Ophthalmol. 2015;43(5):443-8.

80. Gumus K. Topical coenzyme Q10 eye drops as an adjuvant treatment in challenging refractory corneal ulcers: a case series and literature review. Eye Contact Lens. 2017;43(2):73-80. https:// doi.org/10.1097/ICL.0000000000000229.

81. Wang A, Weinlander E, Metcalf B. Use of topical insulin to treat refractory neurotrophic corneal ulcers. Cornea. 2017;36:1426-8.

82. Chen D, Frizzi K, LS G. Repeated monitoring of corneal nerves by confocal microscopy as an index of peripheral neuropathy in type-1 diabetic rodents and the effects of topical insulin. J Peripher Nerv Syst. 2013;18:306-15.

83. Shanley L, McCaig C, Forrester J. Insulin, not leptin, promotes in vitro cell migration to heal monolayer wounds in human corneal epithelium. Invest Ophthalmol Vis Sci. 2004;45:1088-94.

84. Hussar D, Cheeseman R. Lifitegrast (Xiidra) for dry eye disease. J Am Pharm Assoc. 2017;57(2):284-7.

85. Zhang Y, Chen P, Di G, Qi X, Zhou Q, Gao H. Netrin-1 promotes diabetic corneal wound healing through molecular mechanisms mediated via the adenosine 2B receptor. Sci Rep. 2018;8(1):1-2.

86. Han Y, Jiang N, Su T, Yang QC, Yan CC, Ye L, et al. Netrin-1 promotes epithelium repair in corneal injury. Int $\mathrm{J}$ Ophthalmol. 2020;13(2):206-12.

87. Chakrabarty K, Shetty R, Ghosh A. Corneal cell therapy: with iPSCs, it is no more a far-sight. Stem Cell Res Ther. 2018;9(1):287.

88. Koaik M, Baig K. Corneal neurotization. Curr Opin Ophthalmol. 2019;30(4):292-8.

89. Rönkkö H, Göransson H, Taskinen H. Comparison of peripheral nerve regeneration with side-to-side, end-to-side, and end-to-end repairs: an experimental study. Plast Reconstr Surg Glob Open. 2016;4:e1179.

90. Konofaos P, Ver HJ. Nerve repair by means of tubulization: past, present, future. J Reconstr Microsurg. 2013;29(3):149-64.

91. Yoon K, You I, Im S, Jeong T, Park Y, Choi J. Application of umbilical cord serum eyedrops for the treatment of neurotrophic keratitis. Ophthalmology. 2007;114(9):1637-42. 
92. Yanai R, Nishida T, Chikama T, Morishige N, Yamada N, Sonoda K. Potential new modes of treatment of neurotrophic keratopathy. Cornea. 2015;34(Suppl 11):S121-7.

93. Nishida T, Chikama T, Morishige N, Yanai R, Yamada N, Saito J. Persistent epithelial defects due to neurotrophic keratopathy treated with a substance p-derived peptide and insuline-like growht factor 1. Jpn J Ophthalmol. 2007;51(6):442-7.

94. Aifa A, Gueudry J, Portmann A, Delcampe A, Muraine M. Topical treatment with a new matrix therapy agent (RGTA) for the treatment of corneal neurotrophic ulcers. Invest Ophthalmol Vis Sci. 2012;53(13):8181-5.

95. Arvola RPJ, Robciuc A, Holopainen JM. Matrix regeneration therapy : a case series of corneal neurotrophic ulcers. Cornea. 2016;35(4):451-5.

Publisher's Note Springer Nature remains neutral with regard to jurisdictional claims in published maps and institutional affiliations. 